\title{
MiR-20a-5p represses the multi-drug resistance of osteosarcoma by targeting the SDC2 gene
}

Fangfang Zhao ${ }^{1 \dagger}$, Youguang $\mathrm{Pu}^{1 \dagger}$, Mingda Cui ${ }^{2 \dagger}$, Haiyan Wang ${ }^{3}$ and Shanbao Cai ${ }^{1,2,4^{*}}$

\begin{abstract}
Background: As one of the hallmarks of cancer, chemoresistance hinders curative cancer chemotherapy in osteosarcoma (OS). MicroRNAs (miRNAs) act as key regulators of gene expression in diverse biological processes including the multi-chemoresistance of cancers.

Methods: Based on the CCK8 experiments, we performed an RNA-seq-based miR-omic analysis of osteosarcoma (OS) cells (a multi-chemosensitive OS cell line G-292 and a multi-chemoresistant OS cell line SJSA-1) to detect the levels of miR-20a-5p. We predicted Homo sapiens syndecan 2 (SDC2) as one of the target genes of miR-20a-5p via several websites, which was further validated by detecting their expression of both mRNA and protein level in both the miR-20a-5p-mimic transfected G-292 and miR-20a-5p-antagomiR transfected SJSA-1 cells. The involvement of SDC2 with OS chemoresistance was checked by siRNA-mediated repression or overexpression of SDC2 gene. Cell viability was assessed by CCK8 assay.
\end{abstract}

Results: We found that the miR-20a-5p level was higher in G-292 cells than in SJSA-1 cells. Forced expression of miR$20 a-5 p$ counteracted OS chemoresistance in both cell culture and tumor xenografts in nude mice. As one of miR-20a$5 p$ 's targets, SDC2 was found to mediate the miR-20a-5p-induced repression of OS chemoresistance.

Conclusions: Our results suggest that miR-20a-5p and SDC2 contribute to OS chemoresistance. The key players in the miR-20a-5p/SDC2 axis may be a potential diagnostic biomarker and therapeutic target for OS patients.

Keywords: Osteosarcoma, Chemoresistance, miR-20a-5p, SDC2

\section{Background}

MiRNAs are a class of small non-coding regulatory RNA molecules that have been shown to be involved in a wide range of biological processes [1]. Their dysregulation has been associated with the development of diseases including cancer. The abnormal expression of miRNAs in cancer contributes to every aspect of tumor biology $[2,3]$, including drug resistance [4], which remains a major obstacle to effective treatment in patients [5]. As

\footnotetext{
*Correspondence: sbc651116@163.com

${ }^{\dagger}$ Fangfang Zhao, Youguang Pu and Mingda Cui contributed equally to this work

${ }^{1}$ Cancer Epigenetics Program, Anhui Cancer Hospital, West Branch of Anhui Provincial Hospital, Anhui Medical University, Hefei 230031, Anhui, China

Full list of author information is available at the end of the article
}

a hallmark of cancer, the multi-chemoresistance varies drastically among the cancer patients, the different cancer lesions or different regions of the same lesions within a single patient [6]. Despite years of intensive efforts, our knowledge of the cancer multi-chemoresistance remains very limited $[7,8]$. To date, much effort has been exerted in analyzing the role of miRNAs in the development of chemoresistance in a variety of cancers. Several studies have shown that miRNA misregulation can increase chemoresistance in cancer cells if specific proteins are affected [9-11]. For instance, miR-33a was found to be upregulated in osteosarcoma (OS) chemoresistance and to promote resistance to cisplatin by downregulating TWIST [12].

As one of the well-studied miRNAs, miR-20a, a member of the miR-17-92 cluster, has been shown to function 
as an oncomir in many human cancers, including lung cancer [13], hepatocellular carcinoma [14], and gastric cancer [15]. In addition, miR-20, Rest and Wnt signaling is suggested to be involved in a regulatory circuit that can modulate the neural differentiation of neural progenitor cells [16]. It was also found that miR-20a induces cell radio-resistance by activating the PTEN/PI3K/Akt signaling pathway in hepatocellular carcinoma [17].

OS is the most common malignant primary bone tumor in children and adolescents $[18,19]$, and the mechanism for the OS chemoresistance remains largely unknown. In the present study, we performed a RNA-seq based-omic analysis for the differentially expressed genes in a multichemosensitive (G-292) versus a resistant (SJSA-1) OS cell lines. We showed here that miR-20a-5p represses the OS multi-chemoresistance via its down-regulation of the SDC2 gene, a newly identified target of miR-20a-5p.

\section{Materials and methods}

\section{Cell lines and culture}

Two osteosarcoma cell lines used in this study-G-292 (ATCC NO. CRL-1423) and SJSA-1 (ATCC NO. CRL2098) were purchased from the ATCC (https://www. atcc.org/). The two cell lines were cultured in Dulbecco's modified Eagle's medium (Invitrogen, Carlsbad, CA, USA) supplemented with $10 \%$ fetal bovine serum (Invitrogen) and $1 \%$ glutamine at $37{ }^{\circ} \mathrm{C}$ in $5 \% \mathrm{CO}_{2}$.

\section{The mimic/antagomiR/siRNA/overexpression plasmids transfection}

All the mimic, antagomiR, siRNA, and the scramble sequence control (NC) as well as riboFECT CP transfection kits were supplied by Guangzhou Ribobio (Guangzhou, China). The mammalian expression constructs for SDC2 with GFP tag (EX-W2418-M98) were supplied by GeneCopoeia $^{\mathrm{TM}}$ (http://www.genecopoeia.com/). Transfection of both ribonucleic acid reagents or plasmids mentioned above and the reporter plasmids in a Cignal Finder Pathway Reporter package (Qiagen, Hilden, Germany) was performed according to the manufacturer's instruction.

The sequences used in this study are as follows:

si-SDC2:

5'-GAAACCACGACGCTGAATA-3'

hsa-miR-20a-5p

antagomiR: $\quad 5^{\prime}$-CUACCUGCACUAUAAGCACUU

UA-3'

mimic:

sense 5'-UAAAGUGCUUAUAGUGCAGGUAG-3' antisense 5'-CUACCUGCACUAUAAGCACUUUA-3'

\section{The luciferase reporter assay}

Two partial sequences of the human SDC2 3'-untranslated region (276 bp, 1-276 and $533 \mathrm{bp}, 1729-2261$ ) with the miR-20a-5p targeting motif were cloned at the downstream of the firefly luciferase gene in pmiR$\mathrm{RB}^{-R E P O R T} \mathrm{~T}^{\mathrm{TM}}$ to construct pmiR-RB-REPORT ${ }^{\mathrm{TM}}$-lucSDC2-WT1 and pmiR-RB-REPORT ${ }^{\mathrm{TM}}$-luc-SDC2-WT2, respectively. Cells were seeded into 96-well plates at around $1 \times 10^{4}$ cells per well and transfected with a mixture of $50 \mathrm{ng}$ pmiR-RB-REPORT ${ }^{\mathrm{TM}}$-luc-SDC2-WT1/ WT2, 5 ng Renilla plus 5 pmol mimic or scramble control (NC) nucleotides, with the riboFECT CP transfection reagents according to the manufacturer's instruction. Both firefly and Renilla luciferase activities were measured $24 \mathrm{~h}$ after transfection by the Dual-Luciferase Reporter Assay System (Promega) using a Promega GloMax 20/20 luminometer. The relative firefly luciferase activities were normalized with the Renilla luciferase activities as a for transfection efficiency.

\section{Chemotherapeutics}

Clinical grades of the following drugs were used (NCI dictionary of cancer terms, http://www.cancer.gov/dictionary), Dox (Haizheng, Zhejiang, China); Etop (Hengrui, Jiangsu, China); MTX (Lingnan, Guangdong, China) and CDDP (Haosen, Jiangsu, China) [5, 20, 21].

\section{Chemoresistance profiling ( $\mathrm{IC}_{50}$ measurements)}

Cells in the logarithmic phase of growth were seeded in triplicate in 96 -well plates at the density of $0.5 \times 10^{4} /$ well and treated with fourfold serially diluted drugs for $72 \mathrm{~h}$. Cell survival was then measured by a thiazolyl blue tetrazolium bromide (CCK8, $450 \mathrm{~nm}$ reading)-based cell proliferation assay [5]. Both the linear regression parameters and the $\mathrm{IC}_{50}$ (the concentration of drug required for $50 \%$ of cells to be killed) with the no-drug control as the reference were calculated. The relative chemoresistance was presented as the fold for each of the cell line over the lowest $\mathrm{IC}_{50}[22]$.

\section{Apoptosis analysis}

Cells were harvested and rinsed with PBS twice. Then, $3 \mu \mathrm{l}$ of FITC-labeled enhanced annexinV and $3 \mu \mathrm{l}(20 \mu \mathrm{g} /$ $\mathrm{ml}$ ) of propidium iodide were added to $100 \mu \mathrm{l}$ of cell suspension. After incubation in the dark for $15 \mathrm{~min}$ at room temperature, the samples were diluted with $100 \mu \mathrm{l}$ of PBS. Flow cytometry was performed on a FACSCalibur instrument. The results were analyzed according to the manufacturer's instructions. The experiments were performed independently three times, and a representative is shown. 


\section{In vitro invasion assays}

Cell invasion assays were performed in a 24-well plate with $8 \mathrm{~mm}$ pore size chamber inserts (Corning, USA). For invasion assays, $1 \times 10^{4}$ cells stably expressing mimic, antagomiR or NC were placed into the upper chamber in each well with the matrigel-coated membrane, which was diluted in serum-free culture medium. In the assay, cells were suspended in $100 \mu \mathrm{l}$ of DMEM without FBS when they were seeded into the upper chamber. In the lower chamber, $500 \mu \mathrm{l}$ of DMEM supplemented with $10 \%$ FBS was added. After incubation for $30 \mathrm{~h}$ at $37^{\circ} \mathrm{C}$ and $5 \% \mathrm{CO}_{2}$, the membrane inserts were removed from the plate, and non-invading cells were removed from the upper surface of the membrane. Cells that moved to the bottom surface of the chamber were stained with $0.1 \%$ crystal violet for $30 \mathrm{~min}$. The cells were then imaged and counted in at least five random fields using a CKX41 inverted microscope (Olympus, Tokyo, Japan). The assays were conducted three independent times [23].

\section{RNA analysis}

RNA-seq analysis was performed by BGI-Tech of China, and RNA-seq library preparation and sequencing were performed by BGI (Shenzhen, China). Following purification, RNA was fragmented using divalent cations at an elevated temperature, and first-strand cDNA was synthesized using random hexamer primers and Superscript TMIII (Invitrogen ${ }^{\mathrm{TM}}$, Carlsbad, CA, USA). Second-strand cDNA was synthesized using buffer, dNTPs, RNaseH, and DNA polymerase I. Short fragments were purified with a QiaQuick PCR extraction kit (Qiagen) and resolved with EB buffer for end reparation and poly (A) addition. The short fragments were subsequently connected using sequencing adapters. After agarose gel electrophoresis, suitable fragments were used as templates for PCR amplification. During the QC steps, an Agilent 2100 Bioanaylzer and an ABI StepOnePlus Real-Time PCR System were used in quantification and qualification of the sample library. Finally, the library (200-bp insert) was sequenced using Illumina HiSeq2000 (Illumina Inc., San Diego, CA, USA). The single-end library was prepared following the protocol of the IlluminaTruSeq RNA Sample Preparation Kit (Illumina) [24].

Total RNA was isolated from the cells during the logarithmic phase using Trizol (Tiangen Biotech Co., Ltd., Beijing, China). For the mRNA analysis, the cDNA primed by oligo-dT was made with a prime Script RT reagent kit (Tiangen Biotech Co., Ltd., Beijing, China), and the mRNA level of SDC2 was quantified by a duplexqRT-PCR analysis where the TaqMan probes with a different fluorescence for $\beta$-actin (provided by Shing Gene, Shanghai, China) were used in the FTC-3000P PCR instrument (Funglyn Biotech Inc., Canada). Using the $2^{-}$ $\Delta \Delta \mathrm{Ct}$ method, the normalization with the $\beta$-actin level was performed before the relative level of the target genes was compared. The sequences of primers and probes used for the qRT-PCR analysis are as follows:

\section{HSDC2 F: 5'-CCTATTGATGACGATGACTACGC-3' HSDC2 R: 5'-CCTATTGATGACGATGACTACGC-3' HSDC2 probe: \\ 5'-ROX-CCTATTGATGACGATGACTACGC-3' \\ hACTB F: 5'-GCCCATCTACGAGGGGTATG-3' \\ hACTB R: 5'-GAGGTAGTCAGTCAGGTCCCG-3' \\ hACTB probe: \\ 5'-CY5-CCCCCATGCCATCCTGCGTC-3'}

\section{Western blotting}

Cells were lysed with a lysis buffer $(60 \mathrm{mM}$ Tris- $\mathrm{HCl}$, pH 6.8, 2\% SDS, 20\% glycerol, 0.25\% bromophenol blue, $1.25 \% 2$-mercaptoethanol) and heated at $95{ }^{\circ} \mathrm{C}$ for $10 \mathrm{~min}$ before electrophoresis. The protein was separated by $12 \%$ SDS-PAGE and then transferred to polyvinyl difluoride membranes (Millipore, Bedford, MA) by electroblotting. After blocking with 5\% non-fat dry milk, the blots were incubated with primary antibodies (against SDC2 and GAPDH). The target bands were revealed by an enhanced chemiluminescence reaction (Pierce), and the relative density (level) of proteins over the GAPDH band was quantified with the Gel-Pro Analyzer (Media Cybernetics). Anti-SDC2 (YT4490) was purchased from ImmunoWay (http://www.immunoway.com/index.asp) and anti-rabbit IgG (SA00001-2) was purchased from San Ying Biotechnology, China (https://www.ptglab.com/).

\section{In vivo studies}

Animal experiments were performed as previously described [22]. Expressions of SDC2 protein were measured using immunochemical analysis on 5-mm slices of formalin fixed paraffin-embedded tumor xenografts in nude mice. Antigens were retrieved by pretreating dewaxed sections in a microwave oven at $750 \mathrm{~W}$ for 5 min in a citrate buffer ( $\mathrm{pH}$ 6) processed with the Super Sensitive Link-Labeled Detection System (Biogenex, Menarini, Florence, Italy). The enzymatic activities were developed using 3-amino-9-ethylcarbazole (Dako, Milan, Italy) as a chromogenic substrate. Following counterstaining with Mayer hematoxylin (Invitrogen), slides were mounted in aqueous mounting medium (glycergel, Dako). Pictures were taken using a LEICA DM 4000B microscope, while the relative level of each protein was calculated using LEICA software, percentage of the mock over the chemotherapeutic treated tumors was calculated and plotted. 


\section{Statistical analyses}

The data are presented as the mean, and the error bars indicate the S.D. All statistical analyses were performed with GraphPad Prism 5. Two-tailed Student's t test, a one-way analysis of variance or Mann-Whitney $U$ test was used to calculate statistical significance. A $P$ value of $<0.05$ was considered significant.

\section{Results}

\section{SDC2 is a positive regulator of the multi-chemoresistance} of OS

Our previous result suggested that G-292 and SJSA-1 cell lines are the multi-chemosensitive and multi-chemoresistant OS cell lines, respectively [22]. To identify the mechanisms that govern the multi-chemoresistance of OS cells, we performed an RNA-seq-based miR-omic analysis of G-292 and SJSA-1 cells (GEO Accession Number: GSE89930). The results showed that a dozen of miRNAs were differentially expressed in the SJSA-1 and the G-292 cells and miR-20a-5p was selected as one of the studied target miRNAs. Here the expression of miR$20 \mathrm{a}-5 \mathrm{p}$ by miR-omic analysis was 10.50 -fold higher in G-292 cells compared with SJSA-1 cells [25]. We further tested the level of miR-20a-5p in G-292 and SJSA-1 OS cell lines by qRT-PCR. The level of miR-20a-5p in G-292 was 8.27 -fold higher than that in SJSA-1, indicating a higher expression of miR-20a-5p in the sensitive OS cells [25].

A given miRNA usually suppresses the expression of various target genes and thus regulates related pathways. We thus predicted the target genes of miR-20a-5p based on the following websites: TargetScan (http://www. targetscan.org/) and microRNA.org (http://www.microrna.org/microrna/getMirnaForm.do). We subsequently compared the expression pattern of shared predicted mRNAs between G-292 and SJSA-1 cells by the RNAseq based miR-omic analysis (GEO Accession Number: GSE89930). Dozens of genes have been found that differentially expressed in the two cell lines. Among them, the SDC2 gene is one of the most significantly differentiated genes that negatively correlate with the expression of miR-20a-5p. Consequently, the expression level of SDC2 was higher in SJSA-1 than G-292 at both mRNA (RNAseq based miR-omic: 11.07:1.00, and qRT-PCR analysis: 102.82:1.00) and protein level (western blot: 2.17:1.00) (Fig. 1a-c). The higher expression of SDC2 in multichemoresistant cells SJSA-1 suggests that SDC2 is a positive regulator of OS multi-drug resistance.

The SDC2 gene is a direct target of miR-20a-5p in OS cells The miR-20a-5p level was significantly higher in G-292 cells than SJSA-1 cells. We found that SDC2 negatively correlates with the level of miR-20a-5p. To check whether

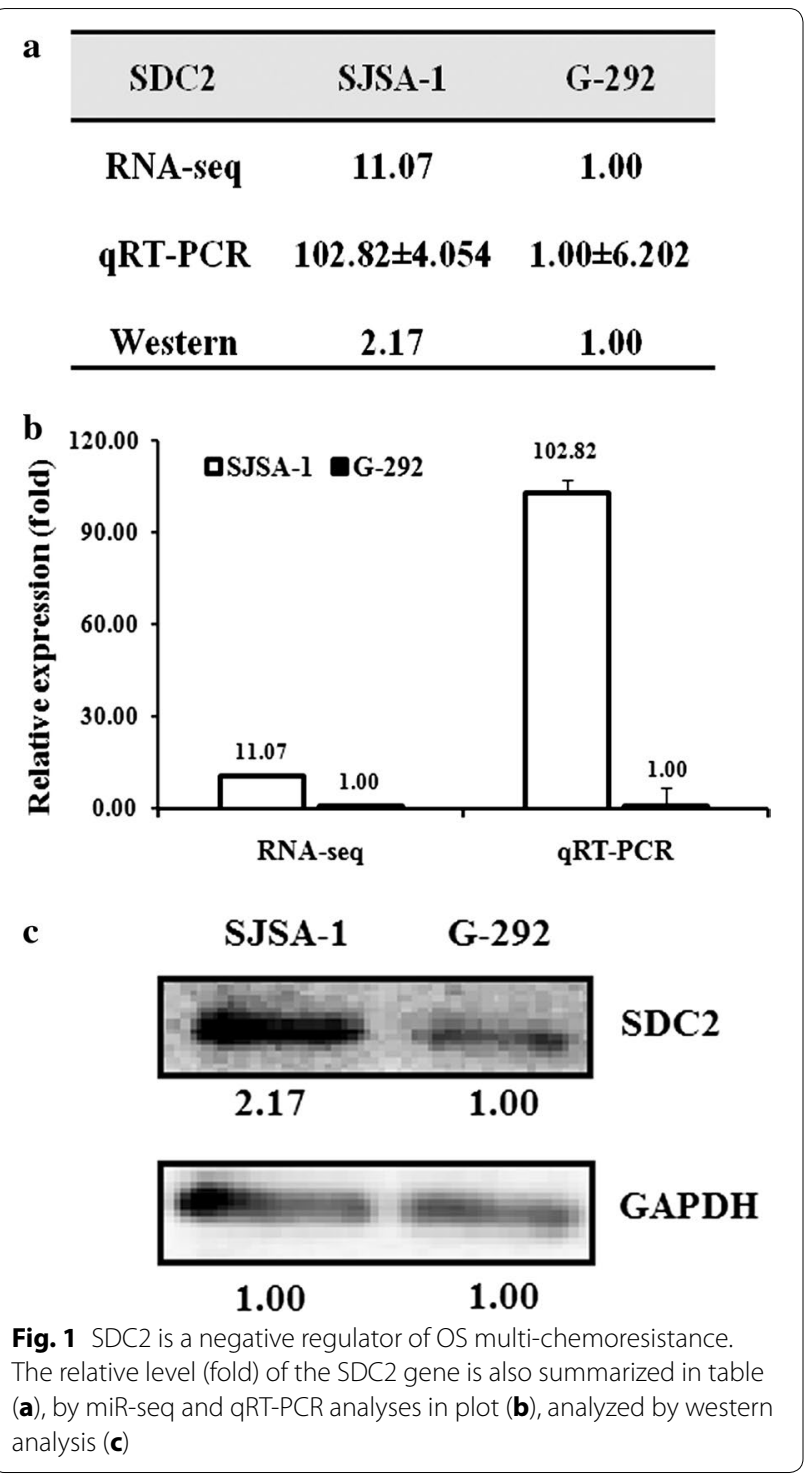

SDC2 is one of the authentic targets of miR-20a-5p, we determined the SDC2 level in the miR-20a-5p mimic transfected SJSA-1 and the antagomiR transfected G-292 cells versus the NC (scramble sequence control) transfected. The transfection of miR-20a-5p mimic in SJSA-1 cells increased its expression to about 39-fold, whereas the transfection of miR-20a-5p antagomiR in G-292 cells significantly decreased its level to $19 \%$ [25]. Following the changes of the miR-20a-5p level, a miR-20a-5p mimic transfection brought down the SDC2 mRNA to 56\% (Fig. 2a) and protein to nearly $20 \%$ (Fig. 2c) compared to that in the NC transfected SJSA-1 cells. As expected, miR-20a-5p antagomiR transfection increased the mRNA level of SDC2 by 2.59 -folds (Fig. 2b) and the protein level by 3.78 -folds in G-292 cells (Fig. 2c). 


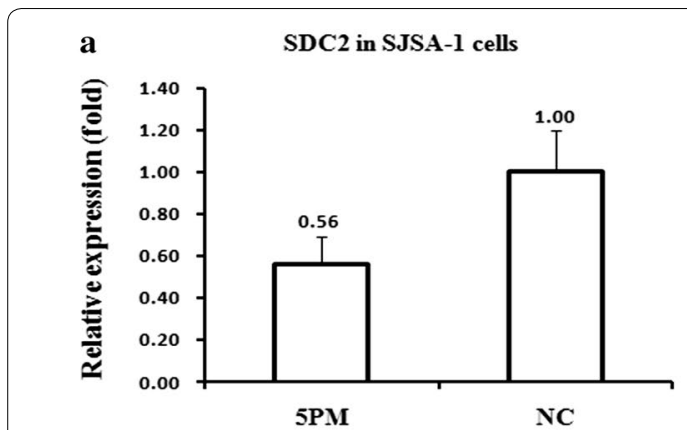

c

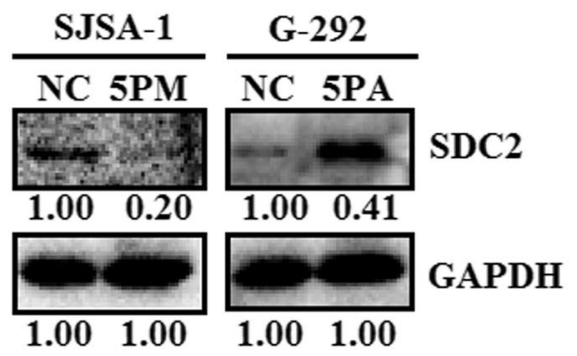

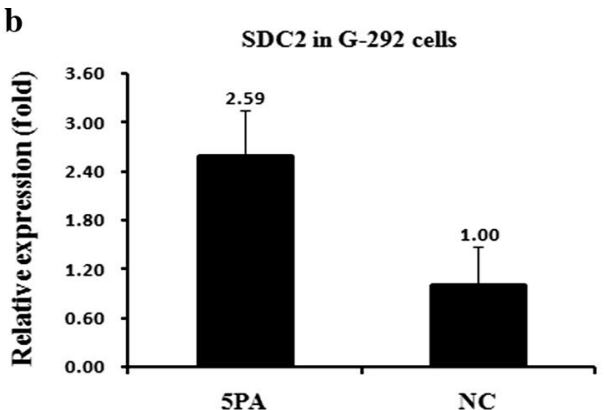

d

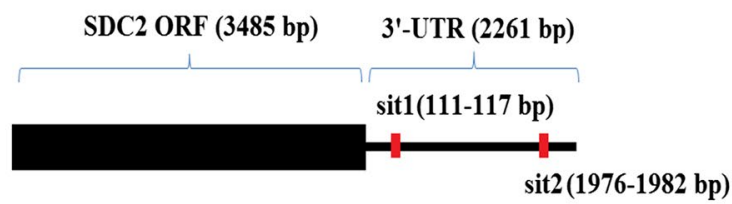

SDC2 3' UTR sit1 WT 5'...UAAAGAGCCAUUCUG GCACUUUA....' hsa-miR-20a-5p 3'-GAUGGACGUGAUAULCGUGAAAU-5' SDC2 3' UTR sit2 WT 5'...CUUACAGAACCAAU UCACUUUA...3' e

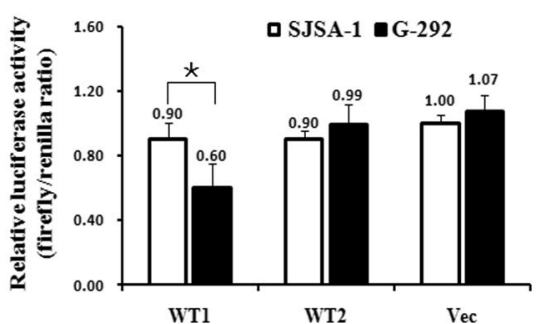

f

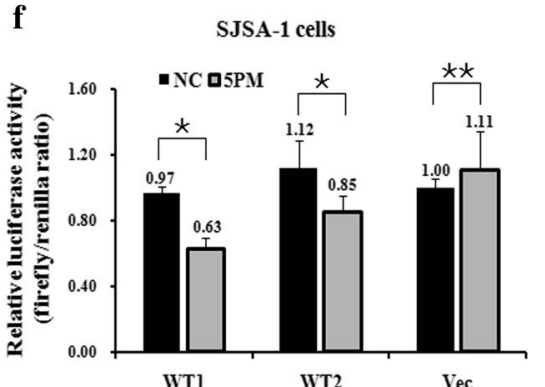

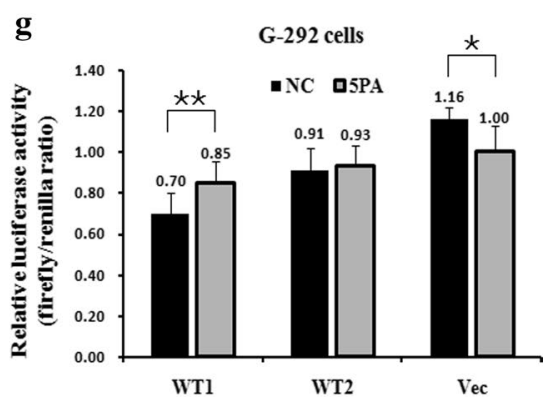

Fig. 2 SDC2 is a target of miR-20a-5p in OS cells. The level of SDC2 mRNA (a, b) and protein (c) in the miR-20a-5p mimic (5PM)-transfected SJSA-1 cell and the miR-20a-5p antagomiR (5PA)-transfected G-292 cell versus the corresponding negative control (NC) determined by western analyses or qRT-PCR. $\mathbf{d}$ The sequences in the UTR region of the SDC2 gene targeted by miR-20a-5p (shaded part). $\mathbf{e}-\mathbf{g}$ The relative luciferase activity (fold) of the reporter with wild-type (WT1 and WT2) SDC2-UTR or with no UTR (Vec) was determined in the miR-20a-5p mimic (in SJSA-1), antagomiR (in G-292) or corresponding mock-transfected OS cells. The Renilla luciferase activity of a co-transfected control plasmid was used to control the transfection efficiency. The representative results from three independent experiments are shown. Error bars represent the s.e.m. ${ }^{*} \mathrm{P}$ value $<0.05$, ${ }^{* *} \mathrm{P}$ value $<0.01$ by Student's $t$ test

Sequence analysis revealed that $3^{\prime}$-UTR region of SDC2 contains two potential binding motifs for miR-20a-5p (termed sit1 and sit2, respectively) (Fig. 2d). To further conclude whether the SDC2 is a target of miR-20a-5p, the wild type 3 '-UTR region of SDC2 was inserted downstream of the luciferase gene in the pmiR-RB-REPORT ${ }^{\mathrm{TM}}$ control vector to create pmiR-RB-REPORT ${ }^{\mathrm{TM}}$-SDC2 UTR WT1 and pmiR-RB-REPORT ${ }^{\mathrm{TM}}$-SDC2 UTR WT2 (Fig. 2e). The constructs pmiR-RB-REPORT ${ }^{\mathrm{TM}}$-SDC2 UTR WT and pmiR-RB-REPORT ${ }^{\mathrm{TM}}$ enhancer control were transfected into G-292 and SJSA-1 cells respectively, to determine whether the differentially expressed
miR-20a-5p in OS cells of distinct chemoresistance is indeed functional. The pmiR-RB-REPORT ${ }^{\mathrm{TM}}$-SDC2-UTR WT1 gave the relative luciferase activities of 0.90 and 0.60 in SJSA-1 and G-292 cells, respectively, and the WT2 was 0.90 and 0.99 respectively (Fig. 2e). The transfection of miR-20a-5p-mimic into SJSA-1 cells significantly brought down the luciferase activity of pmiR-RB-REPORT ${ }^{\mathrm{TM}}$ SDC2-UTR WT constructs, whereas the control cells showed almost the same activity upon the transfection of miR-20a-5p-mimic (Fig. 2f). Meanwhile, the transfection of miR-34a-5p-antagomiR into G-292 cells raised the luciferase activity of pmiR-RB-REPORT ${ }^{\mathrm{TM}}$-SDC2-UTR 
WT constructs (Fig. 2g). It is worthy to notice that sit1 has a more profound effect against the changed level of miR-34a-5p in both G-292 and SJSA-1 cells, which indicated that miR-20a-5p binds more firmly to the sit1 UTR, compared to sit2 UTR. This is also in accordance with the sequence analysis that miR-20a-5p has seven base pairings for sit1 UTR whereas six base pairings for sit2 UTR (Fig. 2d). Getting together, SDC2 is indeed, a direct target of miR-20a-5p and may execute the miR-20a-5p's repressing effect on the OS drug resistance.

\section{The SDC2 expression negatively correlates with the} miR-20a-5p's repressing effect on OS drug resistance

To further demonstrate that miR-20a-5p modulates multi-drug resistance by repressing SDC2 expression in OS cells, we compared drug-triggered cell death in SJSA-1 cells transfected with miR-20a-5p mimic or SDC2 siRNA. The results showed that transfection with the
miR-20a-5p mimic reduced the SDC2 level to $31 \%$ of that found in the NC transfected cells. In addition, SDC2 siRNA inhibited SDC2 protein expression to approximately $45 \%$ of the NC control (Fig. 3a). The transfection of miR-20a-5p mimic or si-SDC2 in SJSA-1 cells decreased the chemoresistance to some extent against the following five drugs: Dox, Etop, MTX, CDDP, Carb, except the mimic to MTX and CDDP (Fig. 3b). Consequently, the transfection of miR-20a-5p mimic or si-SDC2 in SJSA-1 cells showed lower invasion capacity compared to the control cells (Fig. 3c). Afterwards, we increased the level of SDC2 by transfection of miR20a-5p antagomiR or overexpression of SDC2 in G-292 cells. In agreement with the elevated level of SDC2 (Fig. 4a), the cell survival rate was increased for all the five drugs, except the antagomiR to MTX and GFP-SDC2 to CDDP (Fig. 4b). This discrepancy suggested that SDC2 might not mediate OS chemoresistance in response to

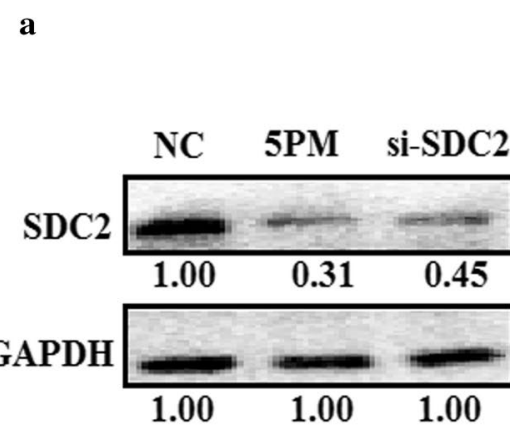

c SJSA-1 cells
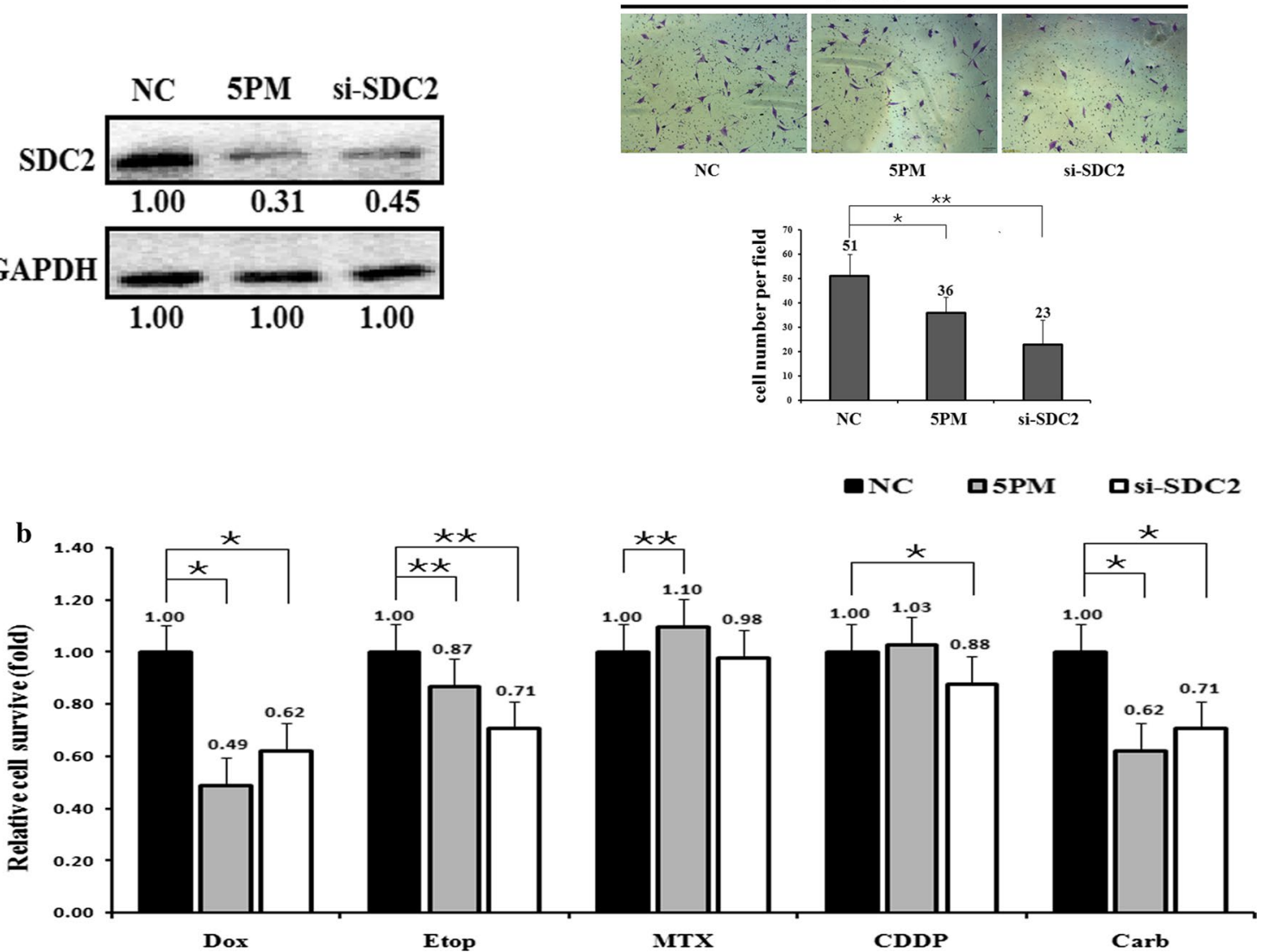

Fig. 3 The effects of a forced reversal of the miR-20a-5p mimic (5PM) or si-SDC2 level on the chemoresistance of SJSA-1 cells. a The SDC2 protein level (western blot analysis) in the miR-20a-5p mimic or siRNA-transfected versus the NC-transfected SJSA-1 cells. b The IC ${ }_{50}$-dosed drug-induced cell death of SJSA-1 cells transfected with the miR-20a-5p mimic (5PM) or si-SDC2 versus the corresponding negative control (NC) assayed $72 \mathrm{~h}$ post-treatment. c MiR-20a-5p mimic or si-SDC2-transfected SJSA-1 cells showed lower invasion capacity compared with the NC-transfected. *P value $<0.05,{ }^{* *} \mathrm{P}$ value $<0.01$ by Student's $t$ test 


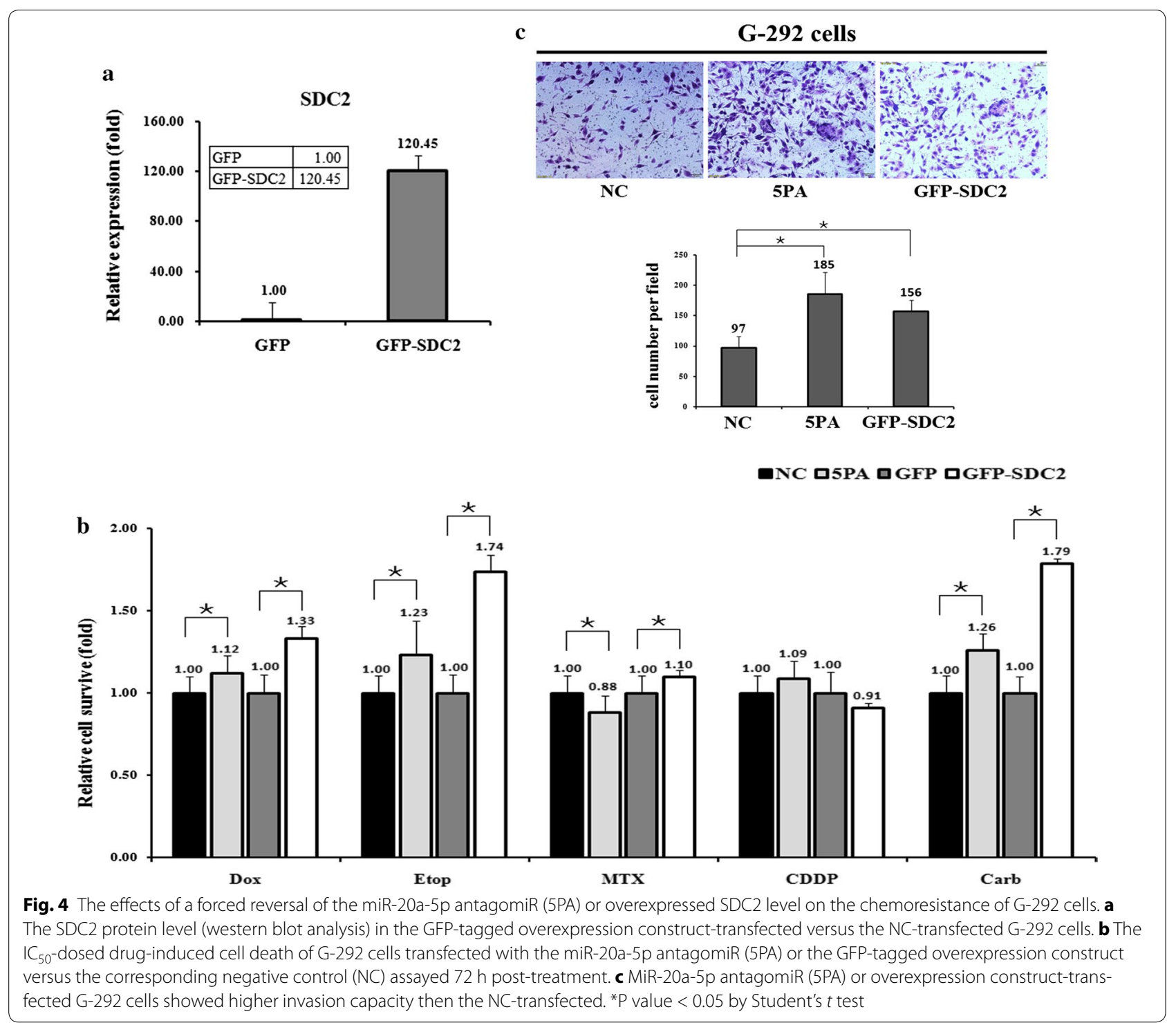

MTX and CDDP. Similarly, the invasion capacity was elevated with the transfection of either miR-20a-5p antagomiR or GFP-SDC2 in G-292 cells (Fig. 4c).

Additionally, the miR-20a-5p mimic mediated SDC2 downregulation in SJSA-1 cells and increased the percentage of apoptotic cells from 0.82 to $0.91 \%$. Similarly, knockdown of SDC2 with siRNA also elevated the apoptotic ratio from 0.59 to $0.87 \%$ in SJSA- 1 cells (Fig. 5a-c). This result indicated that low levels of miR-20a-5p promoted OS cell survival probably by increasing SDC2 expression. All of these observations suggest that SDC2 gene does contribute a great deal to the miR-20a-5p's repressing effect on the OS drug resistance.

\section{MiR-20a-5p suppresses both growth and Dox drug} resistance of the G-292 and SJSA-1-derived tumor xenografts in nude mice

Recently, miR-20a-5p was shown to suppress Dox chemoresistance of OS in tumor xenografts of nude mice via its repression of its target gene KIF26B [25]. In the present study, we semi-quantified via immuno-histological analysis the levels of SDC2 protein in the same set of the tumor tissues in mice that were subjected to an injection of Dox or PBS. The intratumoral injection of miR20a-5p's agomiR into SJSA-1 decreased SDC2 expression. By contrast, the injection of miR-20a-5p's antagomiR into G-292 increased SDC2 expression in Dox- or PBStreated mice (Fig. 6). The results further confirmed that 
a

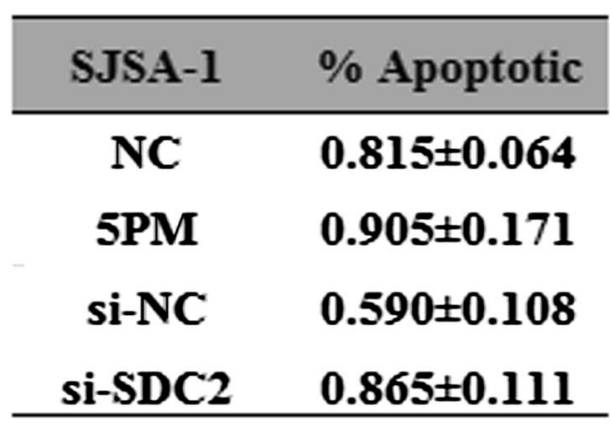

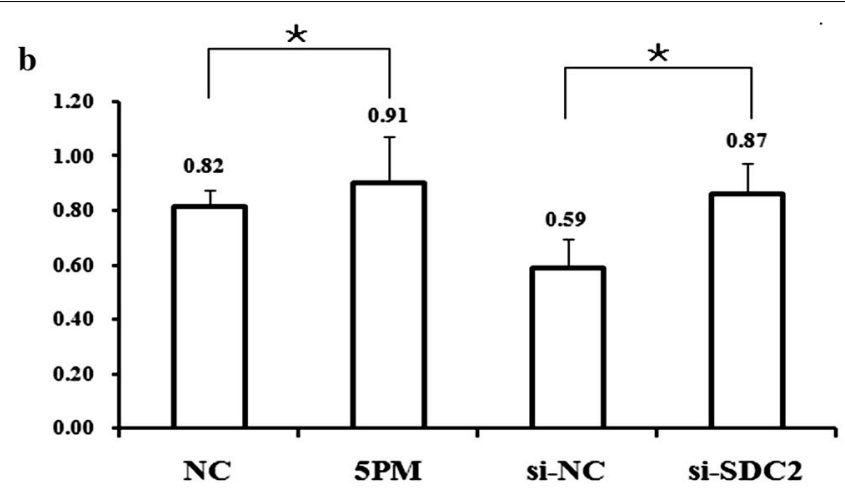

c

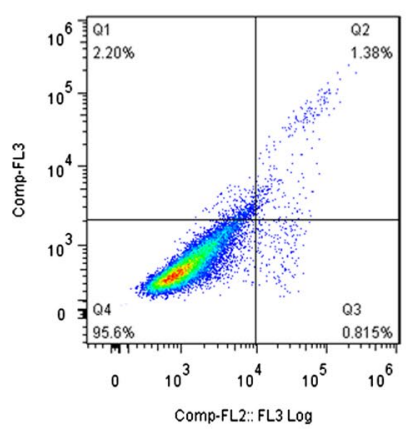

NC

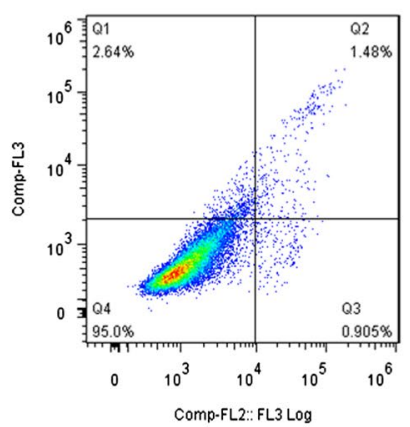

5PM

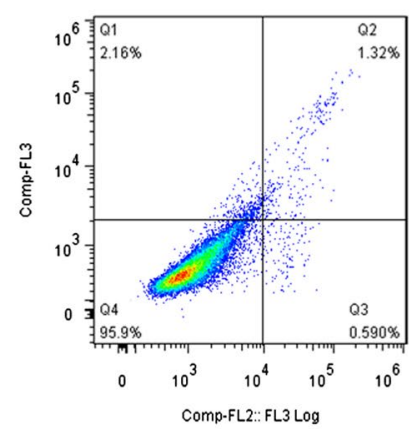

si-NC

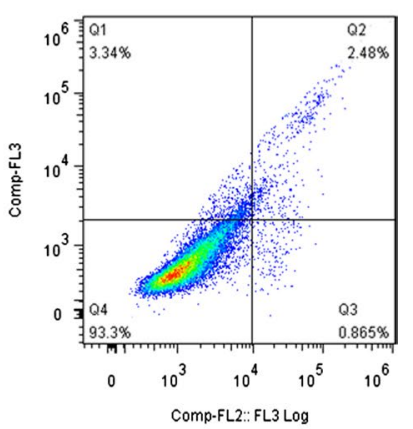

si-SDC2

Fig. 5 Effects of forced alteration of both miR-20a-5p and SDC2 levels on apoptosis in G-292 cells as determined by FACS analysis. *P value $<0.05$ by Student's $t$ test

miR-20a-5p has a profound negative effect on both the growth and chemoresistance of OS cell-derived tumor xenografts in nude mice.

\section{Discussion}

OS chemoresistance is an important topic in the design of clinical treatment protocols because this resistance contributes to relapse and poor prognosis. In this study, we demonstrated that the expression level of miR-20a-5p was elevated in the chemosensitive OS cell line, suggesting that miR-20a-5p might participate in the regulation of OS chemoresistance. MiR-20a-5p expression has been shown to correlate with the development and progression of diverse cancer types [26-32]. For example, miR$20 \mathrm{a}-5 \mathrm{p}$ can be downregulated by glioblastoma hypoxia [31], which often promotes radioresistance and chemoresistance in cancer cells. However, knowledge of the contribution of miR-20a-5p to OS chemoresistance is still limited. In this investigation, we tested the impact of differential expression of miR-20a-5p on cell death in OS cells triggered by commonly used therapeutics.

To explore how miR-20a-5p affects chemoresistance regulation in OS, a luciferase reporter assay was performed to identify potential target genes of miR-20a-5p.
The results showed that miR-20a-5p directly targeted Homo sapiens syndecan 2 (SDC2) in OS cells. SDC2, a cell surface heparan sulfate proteoglycan, is mainly expressed on mesenchymal cells and has been implicated in regulating a broad range of development and disease processes [33-35]. SDC2 exerts various functions in different cell types [36]. For example, SDC2 serves as a coreceptor for fibroblast growth factors and Wnt proteins and controls cell adhesion, proliferation, differentiation and apoptosis [37]. Of note, SDC2 contributes to osteosarcoma cell response to cytotoxic agents through interactions with $\mathrm{Wnt} / \beta$-catenin signaling [38]. Apparently, SDC2 appears to have specific roles in bone cells but the studies of SDC2 effects with respect to tumor chemoresistance are rare. The current study is the first to demonstrate that SDC2 promotes the chemoresistance of OS cells via its upstream regulator miR-20a-5p. In this study, we also showed that SDC2 knockdown in OS cells promoted cell death. These data indicate that suppression of SDC2 inhibits cell survival and further implicate SDC2 as a potential therapeutic target for OS.

In summary, we demonstrated that a miR-20a-5p-centered axis dictates OS multi-chemoresistance. Because of its repressive effect on SDC2, decreasing miR-20a-5p 


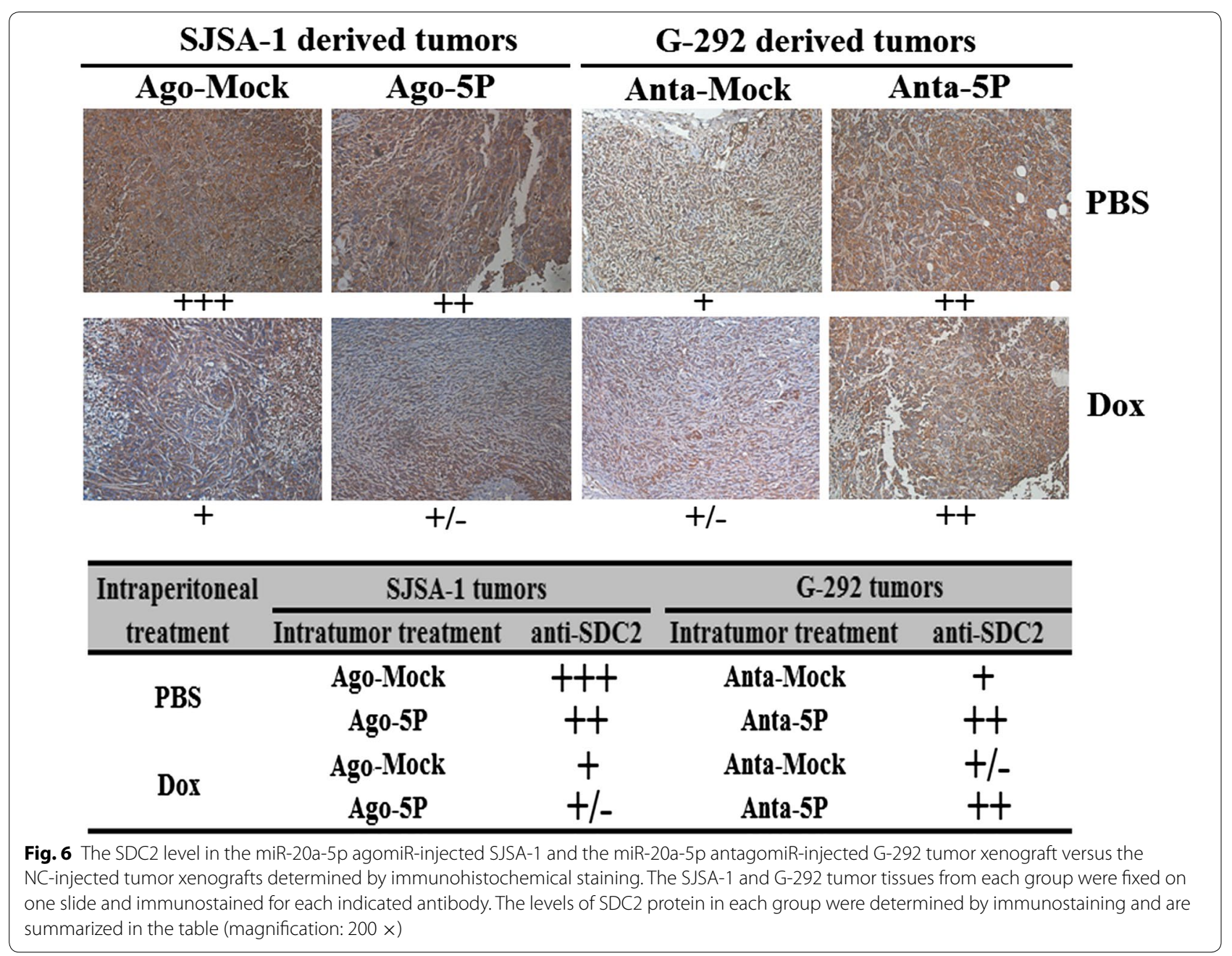

expression promotes OS multi-drug resistance (at least for Dox, Etop and Carb, which were studied in this report) both in vitro and in vivo. Our data suggested that the miR-20a-5p level might serve as a potential biomarker of chemotherapy-resistant OS and that miR20a-5p overexpression might aid in overcoming OS drug resistance.

\section{Conclusions}

In conclusion, this study demonstrated that miR-20a-5p can regulate $\mathrm{OS}$ multi-drug resistance through its direct target gene SDC2 by targeting its $3^{\prime}$-UTR. Our findings suggest that miR-20a-5p may function as a potential candidate for preventing chemoresistance of OS, which may lead to additional new diagnostic and therapeutic approaches for OS and provide new insights into the posttranscriptional regulation of SDC2. Indeed, other regulators of SDC2 may also participate in OS chemoresistance, and our future studies should pay greater attention to examining how SDC2 is regulated in OS or other human tumors.

\section{Abbreviations}

OS: osteosarcoma; MiR: microRNA; UTR: untranslated region; Dox: doxorubicin; Etop: etoposide; CDDP: cisplatin; MTX: methotrexate; SDC2: Homo sapiens syndecan 2.

\section{Authors' contributions}

Conception and design: YGP and SBC. Acquisition of data: YGP, FFZ and HYW. Analysis and interpretation of data: YGP and FFZ. Writing, review, and/or revision of the manuscript: YGP, and SBC. All authors read and approved the final manuscript.

\section{Author details}

${ }^{1}$ Cancer Epigenetics Program, Anhui Cancer Hospital, West Branch of Anhui Provincial Hospital, Anhui Medical University, Hefei 230031, Anhui, China.

2 Xinxiang Medical University, Xinxiang 453003, Henan, China. ${ }^{3}$ Department of Clinical Geriatrics, Anhui Provincial Hospital, Anhui Medical University, Hefei 230031, Anhui, China. ${ }^{4}$ Department of Orthopedic Surgery and Cancer Epigenetics Program, Anhui Cancer Hospital, West Branch of Affiliated Provincial Hospital, Anhui Medical University, Hefei 230031, Anhui, China.

\section{Acknowledgements}

Not applicable. 


\section{Competing interests}

The authors declare that they have no competing interests.

\section{Availability of data and materials}

Not applicable.

\section{Consent for publication}

Not applicable.

\section{Ethics statement}

Animal experiments were undertaken in accordance with the National Institutes of Health Guide for the Care and Use of Laboratory Animals. Animal research was approved by the biomedical ethics committee of Anhui Medical University, when we applying for the National Natural Science Foundation of China (81372868 granted to SBC) in 2013. The animal study proposal was approved by the Institutional Animal Care and Use Committee (IACUC) of the University of Science and Technology of China. All of the mouse experimental procedures were performed in accordance with the Regulations for the Administration of Affairs Concerning Experimental Animals approved by the State Council of People's Republic of China.

\section{Funding}

The National Natural Science Foundation of China (81372868 to SBC) and the Natural Science Foundation of Anhui Province (1408085MH204, $1608085 \mathrm{MH} 224$ and $1608085 \mathrm{MH} 223$ granted to SBC, YGP and HYW, respectively).

\section{Publisher's Note}

Springer Nature remains neutral with regard to jurisdictional claims in published maps and institutional affiliations.

Received: 28 June 2017 Accepted: 24 October 2017

Published online: 02 November 2017

\section{References}

1. Pillai RS, Bhattacharyya SN, Filipowicz W. Repression of protein synthesis by miRNAs: how many mechanisms? Trends Cell Biol. 2007:17(3):118-26.

2. Lu J, Getz G, Miska EA, Alvarez-Saavedra E, Lamb J, Peck D, Sweet-Cordero A, Ebert BL, Mak RH, Ferrando AA, et al. MicroRNA expression profiles classify human cancers. Nature. 2005;435(7043):834-8.

3. Volinia S, Calin GA, Liu CG, Ambs S, Cimmino A, Petrocca F, Visone R, lorio M, Roldo C, Ferracin M, et al. A microRNA expression signature of human solid tumors defines cancer gene targets. Proc Natl Acad Sci USA. 2006;103(7):2257-61.

4. Allen KE, Weiss GJ. Resistance may not be futile: microRNA biomarkers for chemoresistance and potential therapeutics. Mol Cancer Ther. 2010:9(12):3126-36.

5. Lv L, Deng H, Li Y, Zhang C, Liu X, Liu Q, Zhang D, Wang L, Pu Y, Zhang H, et al. The DNA methylation-regulated miR-193a-3p dictates the multichemoresistance of bladder cancer via repression of SRSF2/PLAU/HIC2 expression. Cell Death Dis. 2014;5:e1402.

6. Gerlinger M, Rowan AJ, Horswell S, Larkin J, Endesfelder D, Gronroos E, Martinez P, Matthews N, Stewart A, Tarpey P, et al. Intratumor heterogeneity and branched evolution revealed by multiregion sequencing. New Engl J Med. 2012;366(10):883-92.

7. Marin JJ, Briz O, Monte MJ, Blazquez AG, Macias RI. Genetic variants in genes involved in mechanisms of chemoresistance to anticancer drugs. Curr Cancer Drug Targets. 2012;12(4):402-38.

8. Li F, Sethi G. Targeting transcription factor NF-kappaB to overcome chemoresistance and radioresistance in cancer therapy. Biochem Biophys Acta. 2010;1805(2):167-80.

9. Wang F, Li T, Zhang B, Li H, Wu Q, Yang L, Nie Y, Wu K, Shi Y, Fan D. MicroRNA-19a/b regulates multidrug resistance in human gastric cancer cells by targeting PTEN. Biochem Biophys Res Commun. 2013;434(3):688-94.

10. Bockhorn J, Dalton R, Nwachukwu C, Huang S, Prat A, Yee K, Chang YF Huo D, Wen Y, Swanson KE, et al. MicroRNA-30c inhibits human breast tumour chemotherapy resistance by regulating TWF1 and IL-11. Nat Commun. 2013:4:1393.

11. Zhang Y, Lu Q, Cai X. MicroRNA-106a induces multidrug resistance in gastric cancer by targeting RUNX3. FEBS Lett. 2013;587(18):3069-75.

12. Zhou Y, Huang Z, Wu S, Zang X, Liu M, Shi J. miR-33a is up-regulated in chemoresistant osteosarcoma and promotes osteosarcoma cell resistance to cisplatin by down-regulating TWIST. J Exp Clin Cancer Res. 2014;33:12

13. Hayashita Y, Osada H, Tatematsu Y, Yamada H, Yanagisawa K, Tomida S, Yatabe Y, Kawahara K, Sekido Y, Takahashi T. A polycistronic microRNA cluster, miR-17-92, is overexpressed in human lung cancers and enhances cell proliferation. Cancer Res. 2005;65(21):9628-32.

14. Kutay H, Bai S, Datta J, Motiwala T, Pogribny I, Frankel W, Jacob ST, Ghoshal K. Downregulation of miR-122 in the rodent and human hepatocellular carcinomas. J Cell Biochem. 2006:99(3):671-8.

15. Li X, Zhang Z, Yu M, Li L, Du G, Xiao W, Yang H. Involvement of miR-20a in promoting gastric cancer progression by targeting early growth response 2 (EGR2). Int J Mol Sci. 2013:14(8):16226-39.

16. Cui Y, Han J, Xiao Z, Chen T, Wang B, Chen B, Liu S, Han S, Fang Y, Wei J, et al. The miR-20-Rest-Wnt signaling axis regulates neural progenitor cell differentiation. Sci Rep. 2016;6:23300.

17. Zhang Y, Zheng L, Ding Y, Li Q, Wang R, Liu T, Sun $Q$, Yang H, Peng S, Wang W, et al. MiR-20a induces cell radioresistance by activating the PTEN/PI3K Akt signaling pathway in hepatocellular carcinoma. Int J Radiat Oncol Biol Phys. 2015;92(5):1132-40.

18. Botter SM, Neri D, Fuchs B. Recent advances in osteosarcoma. Curr Opin Pharmacol. 2014:16:15-23.

19. Yang J, Zhang W. New molecular insights into osteosarcoma targeted therapy. Curr Opin Oncol. 2013;25(4):398-406.

20. Heiser LM, Sadanandam A, Kuo WL, Benz SC, Goldstein TC, Ng S, Gibb WJ, Wang NJ, Ziyad S, Tong F, et al. Subtype and pathway specific responses to anticancer compounds in breast cancer. Proc Natl Acad Sci USA. 2012:109(8):2724-9.

21. Andrisano V, Bartolini M, Gotti R, Cavrini V, Felix G. Determination of inhibitors' potency (IC50) by a direct high-performance liquid chromatographic method on an immobilised acetylcholinesterase column. J Chromatogr B Biomed Sci Appl. 2001;753(2):375-83.

22. Pu Y, Zhao F, Wang H, Cai W, Gao J, Li Y, Cai S. MiR-34a-5p promotes the multi-drug resistance of osteosarcoma by targeting the CD117 gene. Oncotarget. 2016;7(19):28420-34.

23. Pu Y, Zhao F, Cai W, Meng X, Li Y, Cai S. MiR-193a-3p and miR-193a-5p suppress the metastasis of human osteosarcoma cells by down-regulating Rab27B and SRR, respectively. Clin Exp Metastasis. 2016;33(4):359-72.

24. Tarazona S, Garcia-Alcalde F, Dopazo J, Ferrer A, Conesa A. Differential expression in RNA-seq: a matter of depth. Genome Res. 2011;21(12):2213-23.

25. Pu Y, Yi Q, Zhao F, Wang H, Cai W, Cai S. MiR-20a-5p represses multi-drug resistance in osteosarcoma by targeting the KIF26B gene. Cancer Cell Int. 2016:16:64

26. Sanfiorenzo C, Ilie MI, Belaid A, Barlesi F, Mouroux J, Marquette $\mathrm{CH}$, Brest $\mathrm{P}$, Hofman P. Two panels of plasma microRNAs as non-invasive biomarkers for prediction of recurrence in resectable NSCLC. PLOS ONE. 2013:8(1):e54596.

27. Xu Q, Dong QG, Sun LP, He CY, Yuan Y. Expression of serum miR-20a-5p, let-7a, and miR-320a and their correlations with pepsinogen in atrophic gastritis and gastric cancer: a case-control study. BMC Clin Pathol. 2013;13:11.

28. Leung CM, Chen TW, Li SC, Ho MR, Hu LY, Liu WS, Wu TT, Hsu PC, Chang HT, Tsai KW. MicroRNA expression profiles in human breast cancer cells after multifraction and single-dose radiation treatment. Oncol Rep. 2014;31(5):2147-56

29. Calvano Filho CM, Calvano-Mendes DC, Carvalho KC, Maciel GA, Ricci MD, Torres AP, Filassi JR, Baracat EC. Triple-negative and luminal A breast tumors: differential expression of miR-18a-5p, miR-17-5p, and miR20a-5p. Tumour Biol. 2014;35(8):7733-41.

30. Ye SB, Li ZL, Luo DH, Huang BJ, Chen YS, Zhang XS, Cui J, Zeng YX, Li J. Tumor-derived exosomes promote tumor progression and T-cell dysfunction through the regulation of enriched exosomal microRNAs in human nasopharyngeal carcinoma. Oncotarget. 2014;5(14):5439-52. 
31. Agrawal R, Pandey P, Jha P, Dwivedi V, Sarkar C, Kulshreshtha R. Hypoxic signature of microRNAs in glioblastoma: insights from small RNA deep sequencing. BMC Genom. 2014;15:686.

32. Honegger A, Schilling D, Bastian S, Sponagel J, Kuryshev V, Sultmann H, Scheffner M, Hoppe-Seyler K, Hoppe-Seyler F. Dependence of intracellular and exosomal microRNAs on viral E6/E7 oncogene expression in HPV-positive tumor cells. PLoS Pathog. 2015;11(3):e1004712.

33. Essner JJ, Chen E, Ekker SC. Syndecan-2. Int J Biochem Cell Biol. 2006;38(2):152-6.

34. Alexopoulou AN, Multhaupt HA, Couchman JR. Syndecans in wound healing, inflammation and vascular biology. Int J Biochem Cell Biol. 2007;39(3):505-28.
35. Tkachenko E, Rhodes JM, Simons M. Syndecans: new kids on the signaling block. Circ Res. 2005;96(5):488-500.

36. Bernfield M, Gotte M, Park PW, Reizes O, Fitzgerald ML, Lincecum J, Zako M. Functions of cell surface heparan sulfate proteoglycans. Annu Rev Biochem. 1999;68:729-77.

37. Mansouri R, Hay E, Marie PJ, Modrowski D. Role of syndecan-2 in osteoblast biology and pathology. BoneKEy Rep. 2015;4:666

38. Dieudonne FX, Marion A, Hay E, Marie PJ, Modrowski D. High Wnt signaling represses the proapoptotic proteoglycan syndecan-2 in osteosarcoma cells. Cancer Res. 2010;70(13):5399-408.

\section{Submit your next manuscript to BioMed Central and we will help you at every step:}

- We accept pre-submission inquiries

- Our selector tool helps you to find the most relevant journal

- We provide round the clock customer support

- Convenient online submission

- Thorough peer review

- Inclusion in PubMed and all major indexing services

- Maximum visibility for your research

Submit your manuscript at www.biomedcentral com/submit 